\title{
The Application of BP Neural Network in Internet of Things
}

\author{
DanDan Cui ${ }^{1, \text { a }}$, Fei Liu $^{2}$ \\ ${ }^{1}$ Software College of Kaifeng University, Henan Kaifeng, 475004, China \\ ${ }^{2}$ Digital Management Center of Kaifeng University, Henan Kaifeng, 475004, China \\ a cuidandankd@163.com
}

Keywords: BP neural network; internet of things; RFID

\begin{abstract}
BP algorithm is a typical artificial neural network learning algorithm, the main structure consists of an input layer, one or more hidden layer, an output layer, the layers of the number of neurons, the output of each node the value is decided by the input values, the role, function and threshold. The Internet of Things is based on the information carrier of the traditional telecommunications network, so that all can be individually addressable ordinary physical objects to achieve the interoperability network. The paper puts forward the application of BP neural network in internet of things. The experiment shows BP is superior to RFID in internet of things.
\end{abstract}

\section{Introduction}

Artificial neural network (based Artificial Neural Network, referred to as ANN) system is to learn from some of the features in the human brain and nervous system to store and process information abstracted from the digital model of an artificial intelligence, with a parallel distributed information processing structure, through the nonlinear function of the compound to approximate the mapping between the input and output. The artificial neural network has many outstanding features, the most good to make decisions on approximate, uncertain, and even contradictions in the knowledge environment, you can solve the artificial design of the weights and the calculation of the correlation coefficient [1]. Neural networks through artificial neural network model of learning can determine the stability of the sample to the model, the dynamic evaluation of the competitiveness of the $\mathrm{C} 2 \mathrm{C}$ electronic retailers and sorting, calculation error, which can guarantee the objectivity of the evaluation results.

Artificial neural network itself contains a lot of network models such as BP neural network, Hopfield network, the Hamming network. Grossberg networks and competitive network, arbitrary precision BP network can approximate any continuous function, so the evaluation of the research literature in the existing competitive BP (Back Propagation network) network mostly. Such as Gao Xiaohong, Guo Jun, Wu Xiaowei (2004) to establish the timing of BP neural network and causal BP neural network prediction of the competitiveness of enterprises, according to the index system of enterprise competitiveness; Li Yuhua (2006) by the BP network on the core competitiveness of the old industrial bases the force was evaluated; Chen transferred (2003) by BP neural network was evaluated on bank competitiveness. These studies evaluate the competitiveness of the BP neural network method is feasible.

Internet of Things is the material objects connected to the Internet. This has two meanings: First, the core and foundation of the Internet of Things is still the Internet is an extension and expansion of Internet-based network; second, between the extension and expansion of its client to any goods and articles, information exchange and communication. Therefore, the definition of things through radio frequency identification (RFID), infrared sensors, global positioning systems, laser scanners, and other sensing devices, according to the agreed protocol, any items with the Internet connection, the exchange of information and communication items intelligent identify, locate, track, monitor and manage a network. The Internet of Things is based on the information carrier of the Internet, the traditional telecommunications network, so that all can be individually addressable ordinary physical 
objects to achieve the interoperability network. It has common object equipment, intelligent autonomy terminal interconnection and universal service three important characteristics. The paper puts forward the application of BP neural network in internet of things.

\section{The neural network of Internet of Things}

BP neural network is the product of artificial intelligence technology developed rapidly and is widely used in various engineering fields, because of its highly nonlinear common in dealing with nature and uncertainty, with a strong nonlinear mapping ability and unique the advantage of much great importance to researchers of various disciplines. So far, it has been widely used in nonlinear modeling, function approximation, pattern classification, etc., is the most widely used neural network model, works well, easy to learn.

Structure and working methods of the artificial neural network according to the neurons in different connections, the neural network are divided into two categories: hierarchical network and interconnected networks layered network all the neurons in a neural network model in accordance with the functions into several layers. General input layer, hidden layer and output layer, each layer sequentially connected. It can also be subdivided into three kinds of interconnect: a simple feedback to the network, prior to before the network layer interconnected to the network. Mutual connection network is any network between the two units is up to, that is, there is a connection path. Interconnected network, it is another branch of the Ministry of interconnection and interconnection. Fully connected network the output of each neuron is connected with other neurons, and local interconnect network between some neural element is not connected relationship.

The Internet of Things refers to the sensing devices through a variety of information, such as sensors, radio frequency identification (RFID) technology, global positioning systems, infrared sensors, laser scanners, gas sensors and other devices and techniques, real-time acquisition of any need to monitor, combine to form a vast network of connections, interactive objects or processes, collecting their sound, light, heat, electricity, mechanics, chemistry, biology, location information of a variety of needs, and the Internet [2]. Its purpose is to achieve things and things, things and people, all of the items connected to the network to facilitate the identification, management and control, as is shown by equation 1 .

$$
\begin{array}{r}
C_{j-1}=H^{\prime} C_{j} H+G^{\prime} D_{j}^{h} H+H^{\prime} D_{j}^{v} G+G^{\prime} D_{j}^{d} G \\
(J=J, J-1, J-2, \cdots, 1)
\end{array}
$$

By storing a large number of input-output models, the nonlinear mapping ability of BP neural network mapping and nonlinear mapping completed by the $\mathrm{n}$-dimensional input space to the m-dimensional output space. Generalization ability in the normal working stage, the neural network training have not seen in normal session, BP neural network has not seen in the training data to complete the correct mapping from input mode to output mode. This is an important factor to measure the BP neural network performance advantages and disadvantages. Fault tolerance to allow the input mode and there is a big error in the samples, and even allows an error.

First, it is widely used for various sensing technology. Things intranet deployment the massive variety of types of sensors, each sensor is a source of information, different types of sensors to capture the information content and information in different formats. The sensor data obtained with real-time, periodic collection of environmental information at a certain frequency, continuous updating of data. Second, it is ubiquitous networks on the Internet. Collected from time to time in the Internet of Things sensor information transmitted over the network, because of its very large number, forming a mass of information, during transmission, must adapt to a variety of heterogeneous networks and protocols, in order to ensure data accuracy and timeliness of it, and is presented by one figure as equation 2 .

$$
q(n)=\frac{\gamma^{-1} R^{-1}(n-1) x(n)}{1+\gamma^{-1} x^{H}(n) R^{-1}(n-1) x(n)}
$$


The Internet of Things is not only the connection of the sensor itself also has the ability of intelligent processing; intelligent control can be implemented on the object. The combination of sensors and intelligent processing of Things, the use of cloud computing, pattern recognition, and other smart technology, expand its application areas. Vast amounts of information from the sensor analysis, processing and handling of meaningful data to accommodate the different needs of different users discover new applications and application mode.

In the hidden layer structure of the research network, compiled using Matlab neural network patch contrast single and double hidden layer structure repair results to determine the repair hidden layers BP neural network; research network training algorithm, the use of visualization the Visual Basic application development tools, the preparation of the application, the large number of experiments to determine the hidden layer nodes, and reasonable network training algorithm is designed to improve the training speed of the network, reducing the network training into local, as is shown by equation3.

$$
\hat{C}_{J}=\left(\alpha C_{J}^{1}+\beta C_{J}^{2}\right) / 2
$$

BP neural network with BP learning, the learning process is divided into four stages: an input mode by the input layer through the hidden layer to the output layer, layer by layer spread pattern along the propagation process. Network desired output the difference between the actual output, ie the error signal, is the correction implied by the output layer by layer to the input layer, layer by layer connection weights of the error back propagation "process." mode along the propagation process and the error back propagation process is repeated alternate network "memory training" process. Networks tend to converge, the global error of the network tend to the minimum, "Learning convergence process.

Internet of Things (Internet of Things) refers to is the ubiquitous (Ubiquitous) terminal equipment (Devices) and facilities (Facilities), including with the "inner intelligence" sensors, mobile terminals, industrial systems, floor control system, the family of Intelligent and "external enable" (enabled), the facilities, video surveillance systems, such as paste RFID a variety of assets (the Assets), and carry the wireless terminal of individuals and vehicles, and other "intelligent objects or animals" or "smart dust"(the Mote), through a variety of wireless / wired long distance / short-range communication networks to achieve interoperability (M2M), application integration (the Grand integration), as is shown by equation 4 .

$$
\left(X_{m}, Z_{i}\right)=\sum_{n=1}^{N} X_{m}(n) Z_{i}^{*}(n)
$$

The paper analyzed the problems of China's real estate valuation market comparison approach, and focus on the AHP market comparison method, fuzzy market comparison approach, the gray market comparison approach, and BP neural network market comparison approach described analysis of the value of its existence and inadequacies. Generalized BP neural network is market comparison method [3]. This method is a combination of principal component analysis and BP neural network will Mathematical Statistics, the use of visualization applications software MATLAB neural network toolbox Programming.

BP network overview of the back-propagation network (Back-Propagation Network, referred to as the BP network) is a nonlinear differentiable function of weights training multi-layer network. BP network is a multi-layer feed-forward neural network, the neuron transfer function is S-shaped function output for 0-1 continuous quantity, it can be any nonlinear from input to output mapping. Due to the adjustment of its weights using back-propagation learning algorithm (Backpropagation), known as the BP network.

Internet of Things is a dynamic global network infrastructure, it has a standards-based and interoperable communication protocols, self-organizing ability, in which physical and virtual "objects" of identity, physical attributes, virtual features, and intelligent interfaces, and seamless 
integration of information networks. Internet of Things Internet and media, the Internet and business Internet one, constitute the future of the Internet, as is shown by equation6.

$$
\left\|Y_{m}\right\|=\left(\sum_{n=1}^{N}\left|Y_{m}(n)\right|^{2}\right)^{1 / 2}
$$

\section{Using BP neural network to build internet of things}

In the design of BP network, the first should be ready data. Matlab7.0 with Excel2003 interlinked, so the survey data to guide into the Matlab7.0. Named is as the "data". Then, $10 \mathrm{C} 2 \mathrm{C}$ electronic retailers competitiveness indicator data as the training input data set to the "traindata"; expert evaluation results of these $10 \mathrm{C} 2 \mathrm{C}$ electronic retailers competitive as the target output is set to "targets; the greater the evaluation value, indicating that this e-retailers more competitive, which constitutes the 10 training samples; the remaining six $\mathrm{C} 2 \mathrm{C}$ electronic retailers competitiveness index measurement data as the simulation data, Make "simulate data. The data are ready; you can build a network, as is shown by equation6.

$$
W=\left(I-\frac{Y_{1}^{*} Y_{1}^{T}}{Y_{1}^{T} Y_{1}^{*}}-\frac{Y_{2}{ }^{*} Y_{2}^{T}}{Y_{2}{ }^{T} Y_{2}^{*}}-\cdots-\frac{Y_{M}{ }^{*} Y_{M}^{T}}{Y_{M}{ }^{T} Y_{M}^{*}}\right) W_{0}
$$

Network input, the number of output neurons is defined by the External Description. So, if there are four external variables as input, then the network there are four inputs. Similarly, if the network has seven outputs, then the output of the network layer should have 7 neurons: Finally, the output signal of the desired features to help you choose the transfer function of the output layer. Research has shown that the two networks use the s-shaped transfer function in hidden layer, linear transfer function in the output layer, almost arbitrary precision approximation of any function of interest, as long as enough units are available in the hidden layer [4].

Summed up the nature of the Internet of Things is mainly reflected in three aspects: First, the Internet features that need to be networked objects must be able to achieve the interoperability of the Internet; identification and communication features, that is included in the Internet of Things "objects" must to have the functions of automatic identification and physical objects communication (BP neural network);According to the actual thesis, designed BP network evaluation model are as follows: A, the input layer: According to the electronic retailers competitiveness evaluation index system, to the lowest level of the number of indicators as the number of input layer neurons, in article 20; B, the hidden layer: hidden layer neuron number selection related to the accuracy and learning efficiency of the BP network, the combination of theoretical analysis and experience in this paper to select hidden layer neurons is 10. Our system runs on a Windows XP-based PC with an Intel Pentium $2 \mathrm{CPU}$ at $2.8 \mathrm{GHz}$. The system is developed using Java on Java SDK 3.4.5 platform, as is shown by figure1.

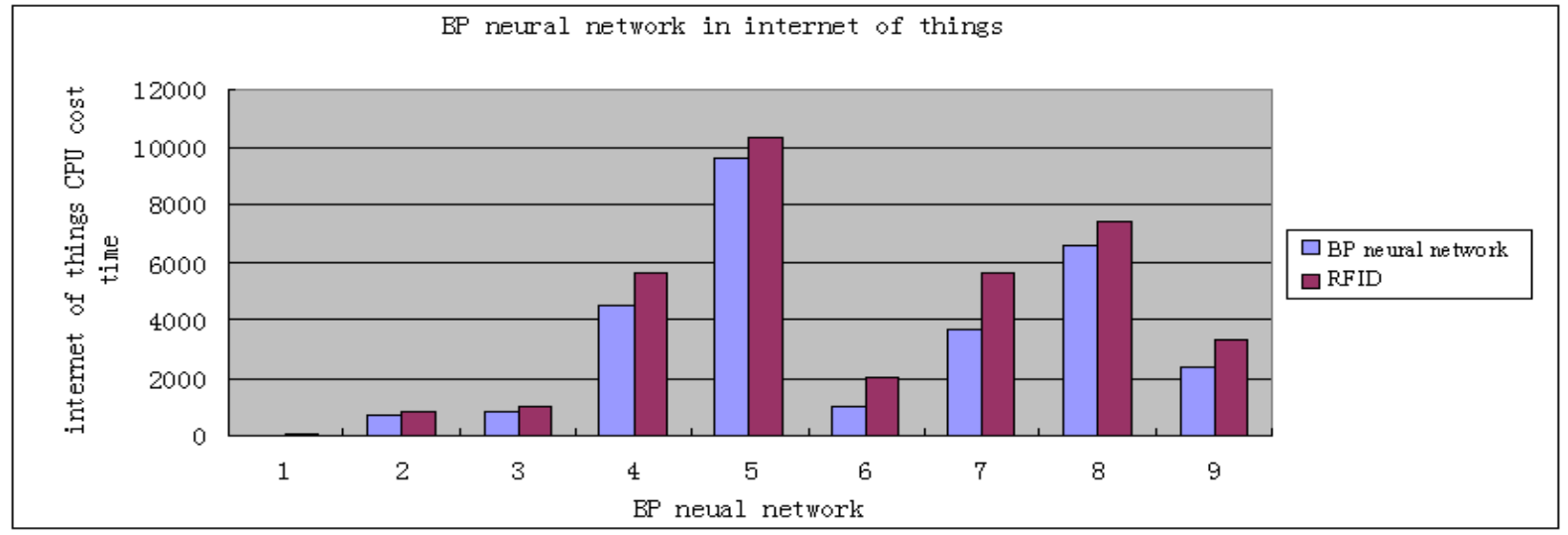

Figure. 1 Development of internet of things compared result of RFID and BP neural network 
The paper puts forward the application of BP neural network in internet of things. In this study, the BP network structure is a layer 2 network with 20-10-1 structure build in matlab7.0.The named bp network, select the type of network Feed-forward backdrop input data range is determined according to the input data in the training sample, the training function to select TRAINLM, adaptive learning function select LEARNGDM, the effect of a function to select the MSE, the number of layers, the first layer10 neurons, the transfer function TANSIG second layer neuron number is 1 , the transfer function for the TANSIG.

BP neural network is also the following main issues: a learning algorithm the slow convergence of BP neural network learning and training too many times, resulting in learning the long training process, learning convergence is too slow, even if it is a relatively simple problem, but also need hundreds or even thousands of times to learn to converge. Existence of local minima of the error function of BP neural network there are multiple local minima, local minima encountered in the network training process can not continue to produce local minima problem.

\section{Summary}

Direct some of the economic benefits of the Internet of Things are mainly concentrated in the Internet of Things related to the field of electronic components, such as radio frequency identification devices, sensors, and so on. The opportunity to bring a huge amount of data transmitted to the network operators, as well as the most downstream impact, such as logistics and retail industries also need quite a long time of observation. The paper puts forward the application of BP neural network in internet of things. BP algorithm is a very effective algorithm, a set of sample input and output into a nonlinear optimization problem, and the use of the most popular gradient descent optimization problems, solving the right to use iterative computation corresponding to learning memory problems, hidden node to the increase in adjustable parameters of the optimization problem, which can get a more accurate solution.

\section{References}

[1] Changsheng Xiang, ZiYing Zhou, "A New Music Classification Method based on BP Neural Network", JDCTA, Vol. 5, No. 6, pp. $85 \sim 94,2011$.

[2] Han Xiao, Yuanjiang Li, "A New Thought based on the Service Composition of Automatic Transmission Semantic Grid in Internet of Things", IJACT, Vol. 3, No. 7, pp. $10 \sim$ 16, 2011

[3] Ren Fang, Ma Jian-Feng, "Attribute-Based Access Control Mechanism for Perceptive Layer of the Internet of Things", JDCTA, Vol. 5, No. 10, pp. $396 \sim$ 403, 2011.

[4] Li xinwu, "A New Color Correction Model for based on BP Neural Network", AISS, Vol. 3, No. 5 , pp. $72 \sim 78,2011$. 